\title{
Modeling the colloidal dispersions: flocculation kinetics though particle dynamics
}

\author{
Vladimir Smirnov* and Evgenij Korolev
}

Moscow State University of Civil Engineering, Yaroslavskoe Shosse, 26, Moscow, 129337, Russia

\begin{abstract}
The problem of stability of colloids recently begins to attract extra attention in construction materials science. This is due to numerous attempts to employ different kind of nanoscale modifiers for production of building materials with enhanced operational properties. Problems of stability and coagulation in colloidal dispersions are studied for several decades, and numerous results were already obtained within framework of Smoluchowski coagulation theory. In the present work we have performed numerical study of the flocculation process and compared the results with well-known ones. It was shown that even for complex pairwise potential the kinetics of number density for isolated particles is not very different from the kinetics which corresponds to constant coagulation kernel. However, for number density of many-particle aggregates we have observed number of peculiarities, including semi-periodic behavior.
\end{abstract}

\section{Introduction}

Problems of stability and coagulation in dispersions are studied since early XX. The importance of these problems is due to the fact that dispersions are widespread both in nature and in technology.

Considering materials science of building materials, it must be noted that both compositions and hardened building materials in most cases are dispersions: the dispersion medium either water or liquid organic matrix material (asphalt, thermoplastic or thermosetting polymer); dispersed phases are hydration products, fine fillers and coarse grained aggregates. Thus, the general problem of stability is quite relevant in construction materials science.

The problem of stability of colloids recently begins to attract extra attention in materials science of building materials [1-3]. This is due to numerous efforts directed to the enhancement of building materials by means of nanotechnology [4-7], including application of modifiers that are based on so-called "primary nanomaterials" - 1D/2D nanoobjects which were synthesized in predefined conditions outside of the composition [8-10]. Such objects can be multi-walled carbon nanotubes, nanoparticles of oxides $-\mathrm{TiO}_{2}$ (in anatase form), $\mathrm{ZnO}$, etc. The goal of nanomodification can only be achieved if the particle is homogeneously distributed and stabilized in form of nano- or micro-suspension.

Design and discovery of materials guided by theory and computation is the current trend in construction materials science [11, 12]. To adequately represent the system with

\footnotetext{
* Corresponding author: smirnov@nocnt.ru
} 
nanoscale objects, it is possible to use particle systems with properly defined pairwise and boundary forces $[13,14]$. Specific form of potentials and numerical values of parameters in their analytical expressions can both be drawn from surface science. There are also numerous results already obtained in the framework of Smoluchowski coagulation theory.

The goal of the present work is to numerically study the flocculation process for model system and to compare the results of numerical experiment with well-known results from coagulation theory.

\section{Prior work}

The stability of colloids can be analyzed from several points of view. Potential of pairwise interaction was derived DLVO $[15,16]$ theory:

$$
U(r)=3 \varepsilon_{0} \varepsilon \kappa \varphi_{d}^{2} e^{-\kappa r}-\frac{A}{6 r} \frac{R_{1} R_{2}}{R_{1}+R_{2}},
$$

where $\varepsilon$ and $\varepsilon_{0}$ are permittivity and vacuum permittivity, $\kappa$ is the inverse screening length, $\varphi_{d}$ is the potential of double layer, $R_{1}$ and $R_{2}$ are radii of spherical particles, $r$ is the distance between particles and $A$ is the Hamaker constant:

$$
A=\frac{\pi^{2} N_{a}^{2} b}{v_{m}}
$$

where $N_{a}$ is the Avogadro constant, $b$ is van der Waals constant and $v_{m}$ is molar volume.

With some assumptions, kinetics of coagulation can be studies without taking into account particle dynamics in form of (1). It was done by M. von Smoluchowski [17]; the obtained equation describes number $N_{n}(t)$ of $n$-particle aggregates

$$
\frac{d}{d t} N_{n}(t)=\frac{1}{2} \sum_{i+j=n} k_{i j} N_{i}(t) N_{j}(t)-N_{n}(t) \sum_{i=1}^{\infty} k_{i n} N_{i}(t)
$$

where $k_{i j}$ is the coagulation kernel.

System (3) can be solved analytically in case of constant $k_{i j}=k=$ const kernel:

$$
N_{n}(t)=N_{0} \frac{t^{n-1}}{(1+\alpha t)^{n+1}}, \alpha=\text { const } ;
$$

thus, the dependencies $N_{n}(t)$ for any $n>1$ are with single maxima.

\section{Modeling method}

Expressions (1) and (2) allows to estimate energies which correspond to repulsion (first term in (1)) and attraction (second term) forces. The estimation can later be used during modeling of particle dynamics at nanoscale. It can be also noted, that adequate results can be obtained even if pairwise potential is modeled by different expression, e.g. by sum of Morse and one-term Mie potential:

$$
U(r)=U_{1}\left(e^{-2 \frac{d-r_{m, 1}}{r_{w, 1}}}-2 e^{-\frac{d-r_{m, 1}}{r_{w, 1}}}\right)-U_{2}\left(1+\left(\frac{d-r_{m, 2}}{r_{w, 2}}\right)^{2}\right)^{-1},
$$


where $U_{1}$ and $U_{2}$ are (approximate) depths of potential wells, $r_{m, 1}$ and $r_{m, 2}$ are (approximate) distances to minima, $r_{w, 1}$ and $r_{w, 2}$ are widths of potential wells and $d$ is the distance between surfaces.

The proposed potential curve (5) is shown on Fig. 1. Thick line corresponds to (5) while thin ones correspond to its first and second terms.

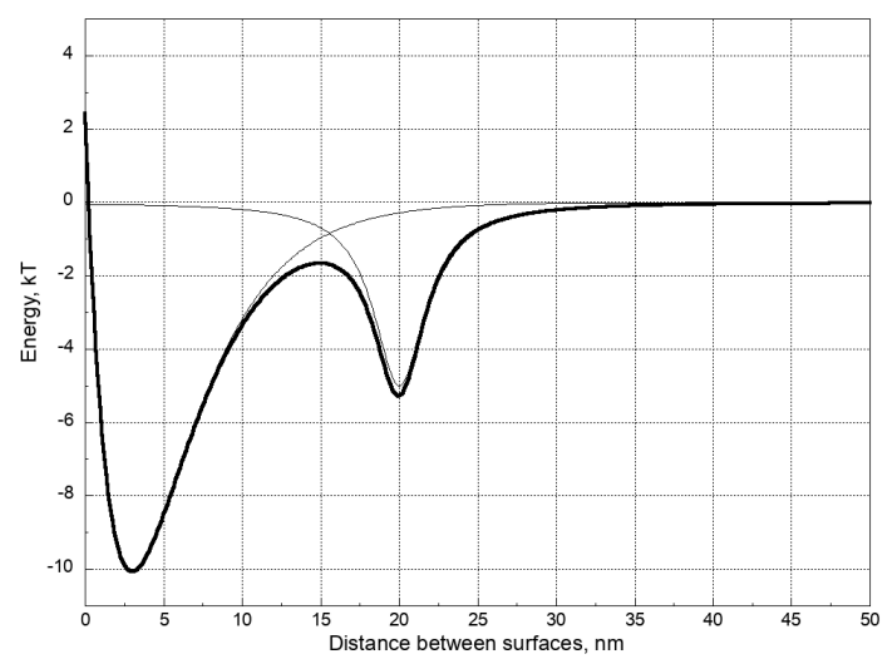

Fig. 1. Potential with two minima suitable for modeling the stability of colloids ( $U_{1}=10 k T$,

$$
\left.r_{m, 1}=3 \mathrm{~nm}, r_{w, 1}=4 \mathrm{~nm}, U_{2}=5 k T, r_{m, 2}=20 \mathrm{~nm}, r_{w, 2}=2 \mathrm{~nm}\right) .
$$

Investigation of flocculation was performed for system with the following parameters:

1. Particle size probability density:

$$
f(R)=0.5 \cdot \delta(R-30 \mathrm{~nm})+0.4 \cdot \delta(R-50 \mathrm{~nm})+0.1 \cdot \delta(R-70 \mathrm{~nm}) .
$$

2. Densities of the particle and dispersive medium are almost equal to each other and are near to $1000 \mathrm{~kg} / \mathrm{m}^{3}$.

3. The viscosity of dispersive medium is non-zero, but set to quite low value to decrease the characteristic times (which otherwise will be six orders of magnitude higher).

4. The values of distances and energies of (7) are set to appropriate values according to DLVO theory.

5. The 512 particles were uniformly (in every coordinate) distributed in spherical domain with radius $5 \mathrm{mkm}$.

For the numerical solution of Cauchy problem we have used the software developed in our university. Design goals and primary features of the software were briefly mentioned earlier $[14,18,19]$.

\section{Results and discussion}

During modeling of the dynamics, several scalar values were registered. Among them:

1. Number $N_{l}$ of particles in largest aggregate (Fig. 2).

2. Average and standard deviation of number $N_{p}$ of neighbor particles - particles which are close than the predefined small distance (Fig. 3).

3. Average distance (and its standard deviation) to four closest neighbors (Fig. 4). 
4. Amount $N_{n}$ of $n$-particle aggregates. For $n=1,2$ and 3 such dependencies are presented on Fig. 5 and 6.

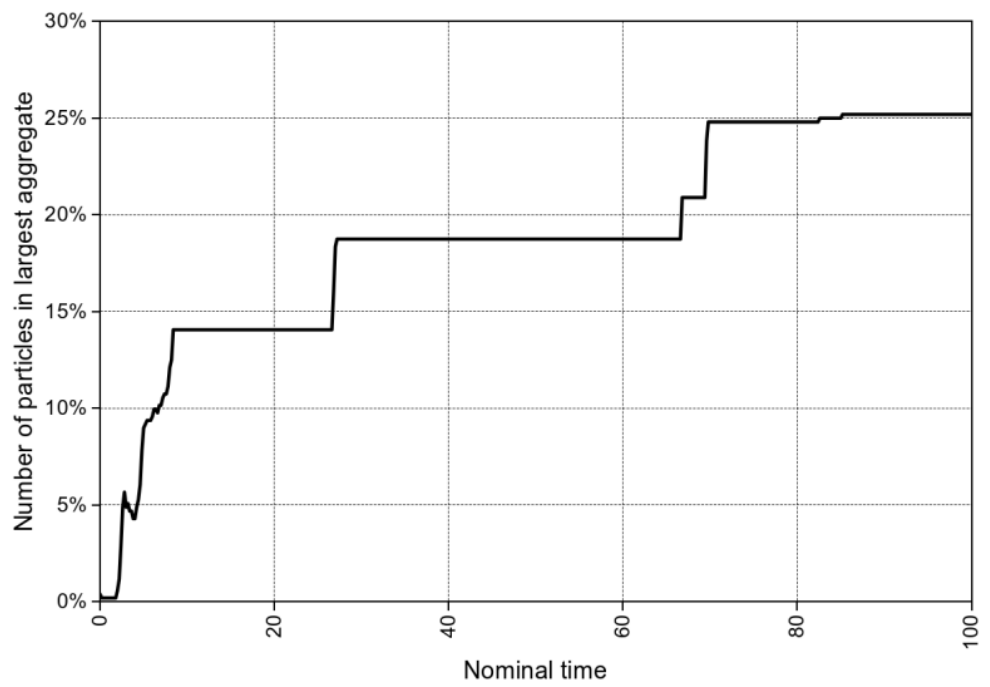

Fig. 2. Number of particles in largest aggregate.

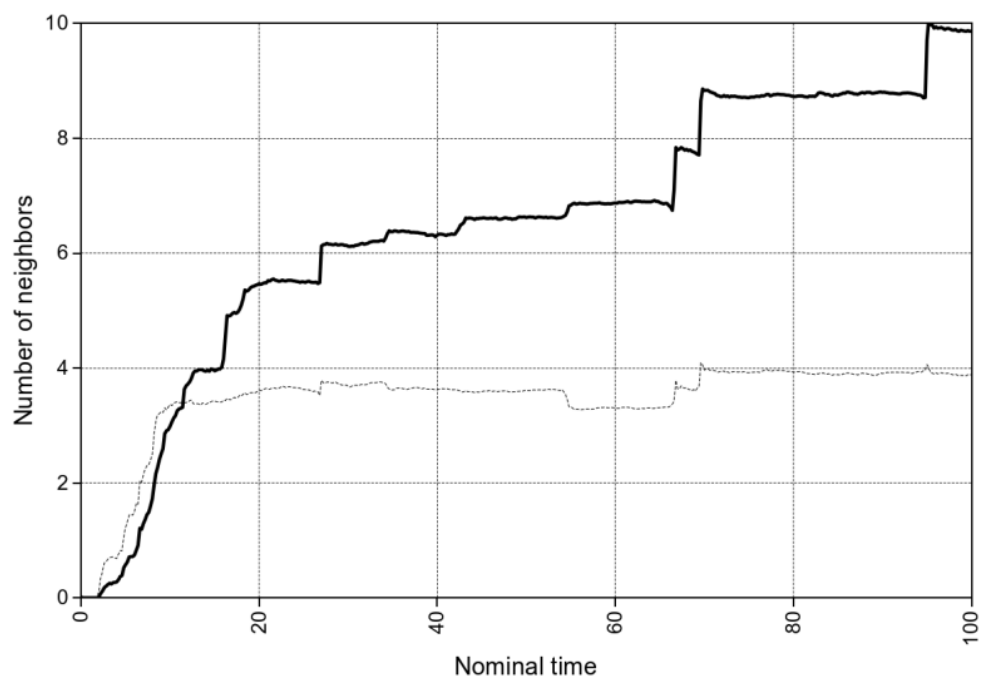

Fig. 3. Average and standard deviation (dashed) of number of neighbors. 


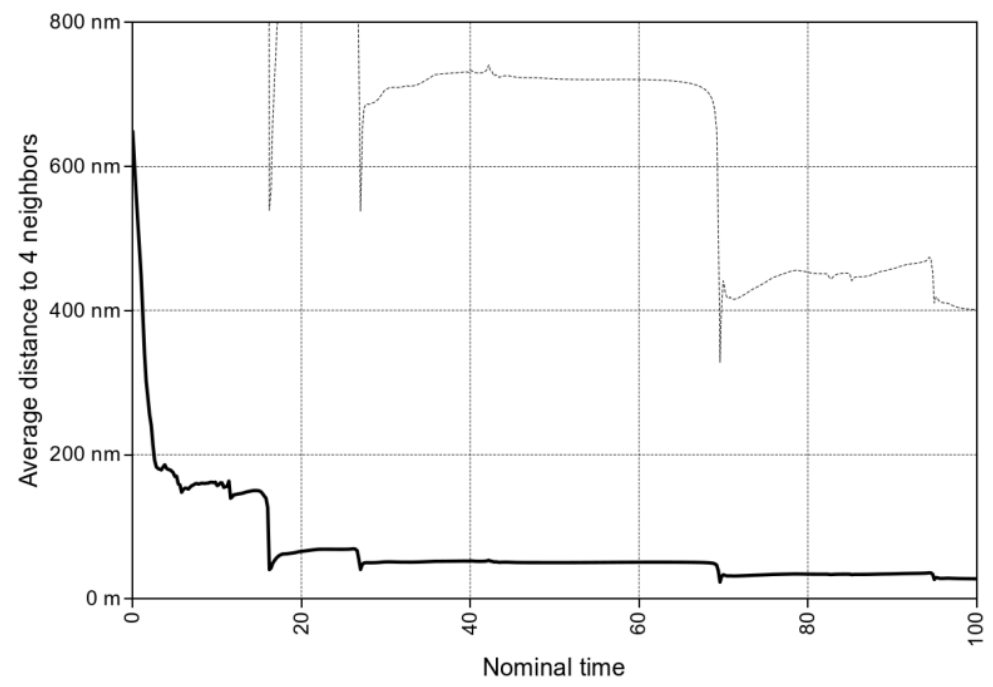

Fig. 4. Average and standard deviation (dashed line) of distance to four closest neighbors.

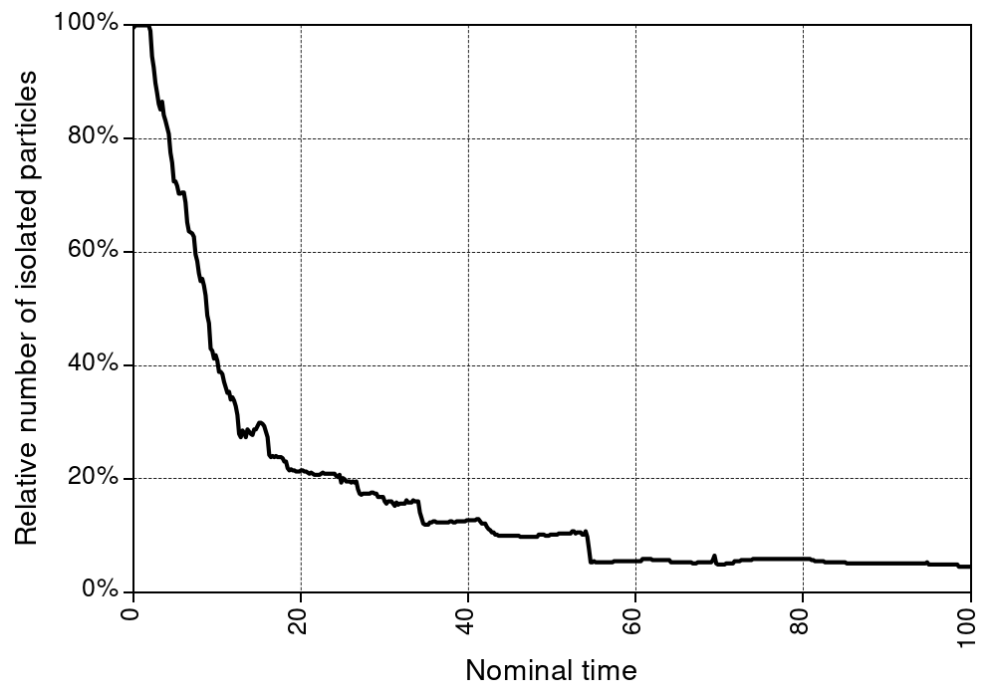

Fig. 5. Number of isolated particles. 


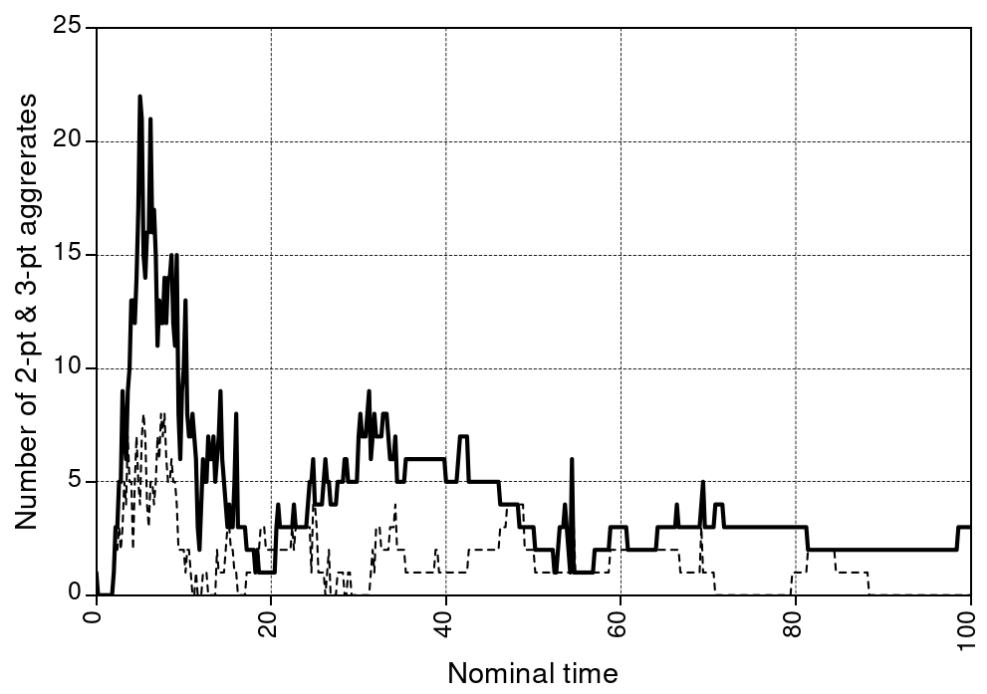

Fig. 6. Number of 2-particle (solid line) and 3-particle (dashed line) aggregates.

As it follows from Fig. 2, with selected parameters of pairwise potential and spatial distribution, all particles are quickly forms several separated aggregates (Fig. 7). The dependence on Fig. 3 indicates dense packing within each aggregate. The low value of standard deviation of $N_{p}$ reflects regular structure of aggregates (Fig. 4, Fig. 8).

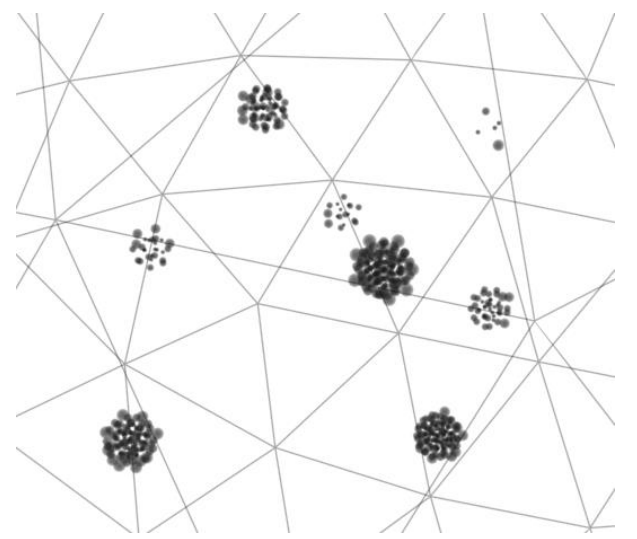

Fig. 7. Final positions of particles.

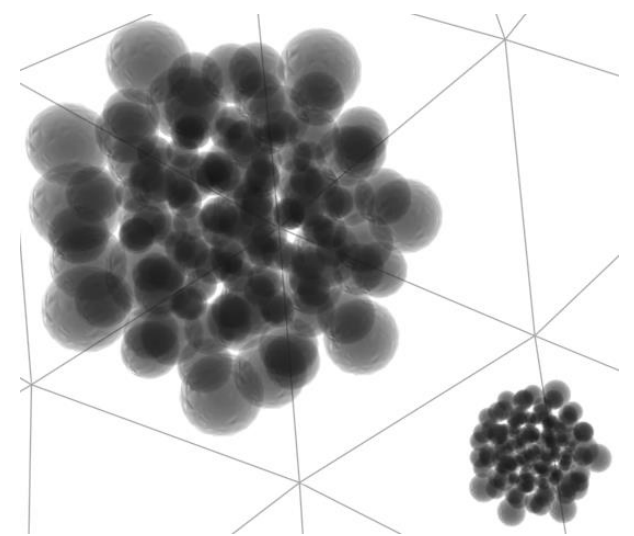

Fig. 8. Aggregates.

The dependence on Fig. 5 corresponds to the solution (4) of Smoluchowski equation for $n=1$ :

$$
N_{1}(t)=\frac{N_{0}}{(1+\alpha t)^{2}} .
$$

Both the curve on Fig. 5 and solution (7) are monotonically decreasing functions whose asymptotic values are zeros. It is possible to apply regression modeling to source data of Fig. 5 and construct the biparametric model:

$$
N(t)=a\left(1+\frac{t}{b}\right)^{-2} .
$$


The obtained parameters are: $a \approx 582, b \approx 17.35$ s. The fitted curve (Fig. 9) is in good correspondence with numerical experiment, though the value of $a$ parameter is a bit higher than the number of particles in system. Thus, Smoluchowski equation with constant coagulation kernel is only an approximate model for the system under consideration.

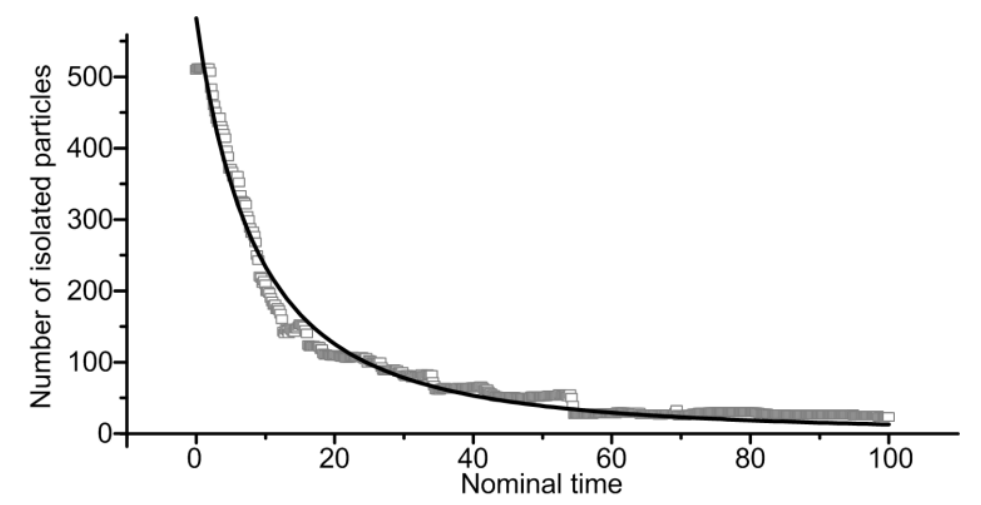

Fig. 9. Fitting $N_{1}(t)$ experimental data with model (8) that is the solution of Smoluchowski equation.

The latter statement is fully confirmed by the dependencies on Fig. 6. While solution (4) predicts one wide maximum and monotonic decrease, the obtained dependencies $N_{2}(t)$ and $N_{3}(t)$ are far from monotonic after their maxima. Moreover, we can observe the similarities in distances between sequential maxima. To highlight the latter peculiarity, the Fourier transforms and frequency spectra of data on Fig. 6 can be calculated. The lowfrequency part of the spectrum for $N_{2}(t)$ is shown on Fig. 10.

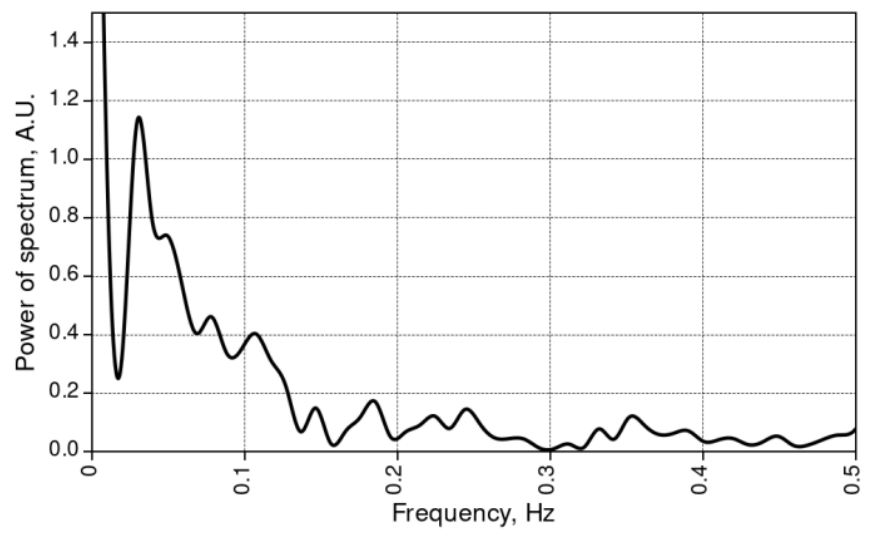

Fig. 10. Frequency spectrum of $N_{2}(t)$.

As it follows from Fig. 10, even for system with only 512 particles, there is a distinctive single maximum near $0.03 \mathrm{~Hz}$ frequency (corresponding period $T_{2} \approx 30 \mathrm{~s}$ ). Thus, Fig. 10 confirms the quasi-periodic character of two-particle aggregate forming. Both reason and consequences of this effect still need exploration. 


\section{Summary and conclusion}

Particle systems are reasonable representations of both compositions and composites in constructional material science [20]. In this work we have presented several novel results of the flocculation modeling. The obtained data allow not only to visualize the spatial configurations, but also to reveal many topological properties of the formed structures and to compare the results with well-known solutions of Smoluchowski equation.

It is shown that for quite complex pairwise potential the kinetics of number density for isolated particles is similar to kinetics for constant coagulation kernel. However, in case multi-particle aggregates, number density changes in complex, semi-periodic manner. This observation still needs further study.

This work is supported by Ministry of Science and Higher Education of Russian Federation, project "Theoretical and experimental models of functional composites based on prime nanomaterials" $7.6250 .2017 / 8.9$

\section{References}

1. S. Ghazizadeh, P. Duffour, N.T. Skipper, M. Billing, Y. Bai, Cement and Concrete Research 99, 116-128 (2017)

2. M.S. Konsta-Gdoutos, Z.S.Metaxa, S.P.Shah, Cement and Concrete Research 40, 1052-1059 (2010)

3. A. Grishina, E. Korolev, E3S Web of Conferences 33, 02035 (2018)

4. K. Sobolev, M.F. Gutiérrez, American Ceramic Society Bulletin 84(11), 16-19 (2014)

5. S. Inozemtsev, E. Korolev, MATEC Web of Conferences 196, 04052 (2018)

6. K. Sobolev, Frontiers of Structural and Civil Engineering 10(2), 131-141 (2016)

7. V. Gladkikh, E. Korolev, V. Smirnov, IOP Conference Series: Earth and Environmental Science 90(1), 012079 (2017)

8. H. Du, H.J. Gao, S.D. Pang, Cement and Concrete Research 83, 114-123 (2016)

9. I. Flores-Vivian, R.G.K. Pradoto, M. Moini, M. Kozhukhova, V. Potapov, K. Sobolev, Frontiers of Structural and Civil Engineering 11(4), 436-445 (2017)

10. S. Yeşilmen, Y. Al-Najjar, M.H. Balav, M. Şahmaran, G. Yildirim, M. Lachemi, Cement and Concrete Research 76, 170-179 (2015)

11. J.S. Dolado, K. Breugel, Cement and Concrete Research 41, 711-726 (2011)

12. A. Kendall, G.A. Keoleian, M.D. Lepech, Materials \& Structures 6, 1117-1231 (2008)

13. F. Sanchez, L. Zhang, J. Colloid Interface Sci., 323, 349-358 (2008)

14. E.V. Korolev, V.A. Smirnov, Advanced Materials Research 746, 277-280 (2013)

15. B. Derjaguin, L.D. Landau, Acta Physicochimica U.R.S.S. 14, 633-662 (1941)

16. F. Family, D.P. Landau (eds.), Kinetics of Aggregation and Gelation (North Holland, Amsterdam, 1984)

17. M. Smoluchowski, Zeitschrift f. Physik. Chemie 92, 129-168 (1917)

18. V. Smirnov, A. Evstigneev, E. Korolev, MATEC Web of Conferences 106, 03027 (2017)

19. A. Evstigneev, V. Smirnov, E. Korolev, MATEC Web of Conferences 251, 01033 (2018)

20. M. Moini, K. Sobolev, I. Flores-Vivian, A. Amirjanov, Journal of Materials in Civil Engineering 31(3), 04019001 (2019) 